DESY $94-010$

DES4 g4 -010 .

se 9406

KEK 93-179

KEK CP-012

INP MSU 93-30/322

January 1994

PQ0020421

\title{
Single Top Quark Production at LEP200?
}

E. Boos, M. Sachwitz, H. J. Schreiber, S. Shichanin

Deutsches Elektronen-Synchrotron DESY

Institut für Hochenergiephysik IfH, Zeuthen

A. Pukhov

Nuclear Physics Institute, Moscow State University, Russia

V. Ilyin, T. Ishikawa, T. Kaneko, S. Kawabata, Y. Kurihara, Y. Shimizu, H. Tanaka

KEK, Tsukuba, Japan

ISSN 0418-9833 

DESY 94- 010

KEK 93-179

KEK CP-012

INP MSU 93-30/322

January 1994

\title{
Single Top Quark Production at LEP200 ?
}

\author{
E. Boos ${ }^{1}$ M. Sachwitz, H. J. Schreiber, S. Shichanin ${ }^{2}$ \\ DESY-Institute für Hochenergiephysik, Zeuthen, FRG
}

A. Pukhov

Nuclear Physics Institute, Moscow State University, 119899, Moscow, Russia

V. Ilyin ${ }^{1}$, T. Ishikawa, T. Kaneko, S. Kawabata, Y. Kurihara, Y. Shimizu, H. Tanaka

KEK, Tsukuba, Japan

\footnotetext{
${ }^{1}$ On leave of absence from Nuclear Physics Institute, Moscow State University, 119899, Moscow, Russia

${ }^{2}$ On leave of absence from Institute for High Energy Physics, 142284, Protvino, Moscow Region, Russia
} 


\section{Abstract}

A complete tree-level calculation of the reaction $e^{+} e^{-} \longrightarrow e^{+} \nu_{e} \bar{t} b\left(e^{-} \bar{\nu}_{e} t \bar{b}\right)$ in the electroweak standard theory in the LEP200 energy range is presented. For top quark masses in the range 130 to $190 \mathrm{GeV}$ the cross sections are found to be in the order of $10^{-5}$ to $10^{-6} \mathrm{pb}$. Therefore, the number of single top quark events is expected to be negligible even with an integrated luminosity of $\mathcal{L}=500 \mathrm{pb}^{-1}$. It is further demonstrated that the Weizsäcker-Williams approximation is approaching the accurate cross section calculations reasonably well.

\section{Introduction}

The existence of the 6th quark, the top quark, is a direct prediction of the Standard Model (SM) [1]. The search for it and the determination of its properties is one of the most important tasks of present high energy physics. The experimental limit for the mass of the top quark, obtained at the TEVATRON, is $[2]$

$$
m_{\text {top }}>113 \mathrm{GeV}
$$

at $95 \%$ C.L., for a top with standard model decays. In addition, precision electroweak measurements from LEP100, $M_{W}$ determination at hadron colliders and neutrino scattering measurements constrain the top quark mass through radiative corrections to the range [3]

$$
m_{\text {top }}=162_{-17-21}^{+16+18} \mathrm{GeV} \text {. }
$$

There is the hope that by upgrading the TEVATRON a top quark with a mass below $\sim 170 \mathrm{GeV}$ will either be discovered or excluded. The main mechanism of top production in hadron collision is $t \bar{t}$ pair production.

The possibility for single top quark production through $\mathrm{W}$-gluon scattering in hadron collisions has been discussed in several papers (see e.g. [4]).

Single top production in the reaction $e^{+} e^{-} \longrightarrow W t b$ has been studied in ref. [5] for energies in the range of the Next $e^{+} e^{-}$Linear Collider. Taking into account the top mass limits (2), the threshold for this reaction is above the standard LEP200 energy range. In [6] reactions for light top production were considered, while single top production in $\gamma e$ collisions at $\mathrm{TeV}$ energies has been studied in ref. [7]. Single top production might be interesting for measurements of top quark characteristics like the $V_{t b}$ Kobayashi-Maskawa angle [8] or the top width, quantities hardly accessible in $t \bar{t}$ pair production. 
Within the Standard Model, at LEP200 energies single top quark production occurs in the reaction

$$
e^{+} e^{-} \longrightarrow e \nu t b
$$

for $m_{\text {top }}$ values being in the range (2).

Recently results from calculations of reaction (3) have been published [9] with the conclusion that LEP200 would provide a chance to observe top quarks with masses up to $\sim 180 \mathrm{GeV}$. The authors of ref. [10] however concluded that with the anticipated luminosity of LEP200 the expected event rate is very low, even for $\sqrt{s}=200-250 \mathrm{GeV}$. A clarification of the situation seems mandatory; the LEP200 upgrade strategy to reach collision energies of 210 $\mathrm{GeV}$ as suggested in ref. [9] would be influenced by the capability for single top quark production.

In this paper we present the results of complete tree-level calculations for the cross section of reaction (3) within the standard electroweak theory. Throughout our paper, reaction (3) involves beside the $e^{+} \nu_{e} \bar{t} b$ final state also the charge conjugated final state $e^{-} \bar{\nu}_{e} t \bar{b}$. In sect. 2 the precise cross section calculations are presented as functions of $m_{\text {top }}$ for several center of mass (cms) energies. Sect. 3 involves discussions of some delicate features of the calculations which have to be treated in a proper way in order to obtain correct results. In sect. 4 further support for the correctness of our result is obtained from the event rates of reaction (3) as estimated in the framework of the effective photon (Weizsäcker-Williams) approximation. Conclusions are presented in sect. 5.

\section{Complete tree-level cross sections}

In Fig. 1 the complete set of the Feynman diagrams contributing to reaction (3) is presented, except that diagrams with the Higgs boson propagator were omitted because of the very small Yukawa coupling of the Higgs particle to electrons.

The first row involves the $t$-channel photon exchange diagrams. It is expected that these diagrams provide most of the cross section for reaction (3) due to the singular behaviour of the photon propagator at small momentum transfers. The second row of the diagrams corresponds to the $t$-channel $Z^{0}$ boson exchanges. Compared to the photon exchange diagrams one additional diagram occurs here due to the non-zero $Z \nu \bar{\nu}$ coupling. Because of gauge invariance, diagrams with $t$-channel photon and $Z^{0}$ exchanges are closely connected with each other. At large transfer momenta both types of diagrams are comparable in strength and have to be simultaneously considered in any 
calculation. At small $t$, however, the photon-exchange diagrams are dominant and they are, in a good approximation, gauge invariant by themselves. In the following these four photon exchange diagrams are considered as a particular subset which will be denoted as ' 4 diagrams'. The remaining diagrams in Fig. 1 are either photon and $Z^{0}$ s-channel or $t$-channel neutrino and $W$ exchange diagrams.

In this paper we restrict our calculatons to the energy interval of LEP200, namely from 170 to $210 \mathrm{GeV}$, and to top masses between 130 to $190 \mathrm{GeV}$; hence, we are below the thresholds of the reactions $e^{+} e^{-} \longrightarrow W t b$ and $e^{+} e^{-} \longrightarrow t \bar{t}$. Consequently, there are no contributions from on-shell $W$ and/or top quarks so that the width of these particles can be omitted in the calculations. All calculations were carried out with the nominal set of SM parameters, with a $b$-quark mass of $4.7 \mathrm{GeV}$ and the electron mass of $5.1110^{-5} \mathrm{GeV}$. For the fine structure constant the value $1 / 137$ has been taken since most of the cross section is expected to come from photon exchange diagrams (first row in Fig. 1) with very small momentum transfers.

All results were obtained by means of two independent programs, namely CompHEP [11] and GRACE [12]. Feynman diagram generation, matrix element evaluation and the generation of the FORTRAN codes were done automatically by these packages. We emphasize that these packages use complete different approaches in the matrix element evaluation: in GRACE, after the Feynman diagram generation numerical calculations are carried out by means of helicity amplitude algorithms; in CompHEP, the calculations are made in analytical form for squared amplitudes. The use of both programs in parallel gives us great confidence of the correctness of our results, which is important in view of the very large cancellations of contributions from different diagrams due to gauge invariance (see sect. 3).

The integration over the phase space of the 4-body final state was carried out by means of the adaptive Monte Carlo integration package BASES [13]. Different sets of kinematic variables were used to correct elaborations of kinematical singularities in phase space.

For all cases considered the agreement between the results of the two independent programs was very good; deviations turned out to be smaller than $1.5 \%$ for the full set of diagrams and smaller than $0.5 \%$ for the ' 4 diagrams' case. This is in agreement with previous comparisons between CompHEP and GRACE for e.g. $2 \rightarrow 3$ body reactions in $e^{+} e^{-}$collisions at TeV energies [14].

In addition, some calculations were carried out both in the t'Hooft-Feynman as well as in the unitary gauge. The results obtained in these two gauges are again in very good agreement; deviations were less than $0.5 \%$ and consistent 
with the numerical accuracy (see sect. 3).

Fig. 2 presents the total cross section values (solid curve) for the reaction $e^{+} e^{-} \longrightarrow e \nu t b$ as a function of the top quark mass for three cms energies in the LEP200 energy range. A number of cross section values are collected in Table 1 in the column 'full set'.

The most important point of Fig. 2 and Table 1 is, that the cross sections of reaction (3) are very small. In the best case, namely at $\sqrt{s}=210 \mathrm{GeV}$ and $m_{\text {top }}=130 \mathrm{GeV}$, it is $\sigma=4.210^{-5} \mathrm{pb}$ which corresponds, with an integrated luminosity of $\mathcal{L}=500 \mathrm{pb}^{-1}$, to about 0.02 events. Hence, at LEP200 energies single top quark production, although kimematically possible, is completely negligible within the Standard Model. This result contradicts the findings of ref. [9].

\section{Gauge cancellation and other delicate features of the calculations}

One of the technical difficulties in the calculation of cross sections for reaction (3) concerns large cancellations among the contributing diagrams. If we restrict ourself to the four photon exchange diagrams (first row in Fig. 1), their results for the cross sections are shown in Fig. 2 as dashed lines and in Table 1 in column ' 4 diagrams'. As expected, this subset of diagrams represents the complete calculations to a very good approximation. Fig. 3 demontrates the cancellation behaviour among them within the unitary gauge as a function of the cutoff angle between the incoming and outgoing positron. Each diagram squared results to cross sections of $\mathcal{O}\left(10^{1} \sim 10^{2}\right) \mathrm{pb}$, while their coherent sum gives $7.710^{-6} \mathrm{pb}$, for $m_{\text {top }}=140 \mathrm{GeV}$ at $\sqrt{s}=190 \mathrm{GeV}$. Thus, cancellations of the order $\mathcal{O}\left(10^{7}\right)$ are observed for the total cross section. The t'Hooft-Feynman gauge also gives cancellations of the same order of magnitude. Even in cases of an angular cut of $>0.2 \mathrm{mrad}$ between the positrons (as it was necessary in ref. [9] to obtain finite cross section values), the cancellation is still of $\mathcal{O}\left(10^{5}\right)$. Such enormous cancellation of up to $\mathcal{O}\left(10^{8}\right)$ directly influence the accuracy of our numerical calculations. Therefore, we have also calculated cross sections with double precision and quadratic precision variables and found discrepancies to be about $0.5 \%$.

An other important aspect in the cross section calculation concerns the non-zero value of the electron mass. Due to the singular behaviour of the photon propagator total cross sections will be divergent if $m_{e}=0$, while finite numbers are obtained for $m_{e}=5.1110^{-4} \mathrm{GeV}$. The introduction of an angular cut between the positrons helps to avoid the singularity [9], but the cross 
section difference between the two cases can be appreciable.

We have also checked our first-order calculations within the t'Hooft- Feynman as well as the unitary gauge. As an example, at $\sqrt{s}=190 \mathrm{GeV}$ with $m_{\text {top }}$ $=140 \mathrm{GeV}$ we obtained for the total cross section $0.77910^{-6} \mathrm{pb}$ in the unitary gauge and $0.79410^{-6} \mathrm{pb}$ in the t'Hooft-Feynman gauge, which is consistent with the numerical precision of $\sim 0.5 \%$.

In the energy range of LEP 200 and the $m_{\text {top }}$ interval (2), the effective mass of the two-particle systems $\bar{t} b$ and $e^{+} \nu_{e}$ are far from the mass of the on-shell $W$, so that the width of the $W$ (and of the top quark) can to a good approximation be neglected. However, at larger energies reactions like $e^{+} e^{-} \rightarrow W t b$ and $e^{+} e^{-} \rightarrow t \bar{t}$ will be open with sizeable cross sections and our ' 4 diagrams' approximation should be replaced by calculations which take into account all diagrams (see Fig. 1) and their interferences. In this case finite widths of the $W$ and the top have to be introduced in the calculations, which has been found to be a delicate procedure due to gauge invariance [15].

\section{Equivalent photon approximation}

As it has been shown in sect. 3 the four $t$-channel photon exchange diagrams (first row of Fig. 1) represent a very good description of the reaction $e^{+} e^{-} \longrightarrow e \nu t b$ at LEP200 energies. Further confirmation of these results may be obtained by the application of the Weizsäcker-Williams approximation [16] to reaction (3). In this approach the cross section of the $2 \rightarrow 3$ process

$$
\gamma e^{-} \longrightarrow \bar{t} b \nu
$$

with a real photon in the initial state is convoluted with the effective photon spectrum. We used for this spectrum the improved version of ref. [17]:

$$
f(x, \delta)=\frac{\alpha}{2 \pi}\left[\frac{1+(1-x)^{2}}{x} \log \frac{1-x}{x^{2} \delta}-2 \frac{1-x-x^{2} \delta}{x}\right] .
$$

The cross section for reaction (3) we are interested in is then given by

$$
\sigma_{e^{+} e^{-} \rightarrow e^{+} \bar{t} b \nu}(s) \simeq \int_{x_{\min }}^{x_{\max }} \sigma_{\gamma e^{-} \rightarrow \bar{t} b \nu}(x s) f(x, \delta) d x,
$$

where $x$ is the fractional energy transfered by the photon with $x_{\text {min }}=\left(m_{t o p}+\right.$ $\left.m_{b}\right)^{2} / s, x_{\max }=1$, and $\delta=m_{e}^{2} /\left(m_{t o p}+m_{b}\right)^{2}$.

The first term in the improved photon spectrum (5) corresponds to the usual Weizsäcker-Williams approximation which is connected, before the integration 
over the squared momentum transfer $q^{2}$, to $1 / q^{2}$ terms, whereas the second term corresponds to terms of the order $m_{e}^{2} / q^{4}$. The contribution of the second term has been found to be small $(<1 \%)$ and is neglected in our cross section estimations.

Cross sections for reaction (4), calculated in ref.[6] for energies in the $\mathrm{TeV}$ energy range and confirmed by the package CompHEP, were evaluated with CompHEP for different LEP200 energies and top quark masses. The convolution of these results with $f(x, \delta)$ yields the cross sections for reaction (3) as shown in Fig.2 (dotted line) and in Table 1, last column. As can be seen, the cross sections found are again in the order of $10^{-5}$ and $10^{-6} \mathrm{pb}$, so that resonable agreement exists between the Weizsäcker-Williams approximation and the precise calculation as well as the $t$-channel photon exchange diagram approach. The differences are well below $20 \%$.

\section{Conclusions}

Complete tree-level calculations for cross sections within the Standard Model of single top quark production in the reaction $e^{+} e^{-} \longrightarrow e^{+} \nu_{e} \bar{t} b\left(e^{-} \bar{\nu}_{e} t \bar{b}\right)$ are presented. It is found that in the LEP200 energy range of 170 to $210 \mathrm{GeV}$ and for top quark masses between 130 and $190 \mathrm{GeV}$ the cross sections are of the order of $10^{-5}$ to $10^{-6} \mathrm{pb}$, so that the number of single top quark events expected with an integrated luminosity of $500 \mathrm{pb}^{-1}$ is negligible. Hence, the search for and measuring of top quark characteristics as suggested in ref. [9] is out of range of the LEP200 machine even for the best case of $m_{t o p}=130$ $\mathrm{GeV}$ and $\sqrt{s}=210 \mathrm{GeV}$.

We have demonstrated that in the energy range discussed the cross section for the process $e^{+} e^{-} \rightarrow e \nu t b$ can very well be approximated by calculation of the four $t$-channel photon exchange diagrams (first row in Fig. 1). The Weizsäcker-Williams approximation formalism is also able to reproduce the results for this reaction reasonably well.

At higher energies, in particular above the thresholds for the reactions $e^{+} e^{-} \longrightarrow W t b$ and $e^{+} e^{-} \longrightarrow t \bar{t}$, the diagrams in Fig. 1 with on-shell $W$ and top quark production become important. In this case the $t$-channel photon exchange diagrams dominating at LEP200 will only give small corrections. At such high energies, single top quark production is still of interest since direct information on its couplings may be obtained. Further detailed calculations based on all diagrams of Fig. 1 including finite widths for the $W$ and the top quark are required. 


\section{Acknowledgements}

The KEK and NPI MSU groups are indepted to KASUMI Co. Ltd and SECOM Co. Ltd for financial support to the KEK-MSU collaboration. E. B. and S. Sh. would like to thank DESY-IfH Zeuthen and the Zeuthen L3 group for the kind hospitality. V. I. would like to thank KEK for the kind hospitality and fruitful cooperation.

\section{References}

[1] S. L. Glashow, Nucl. Phys. 22 (1961) 579;

S. Weinberg, Phys. Rev. Lett. 19 (1967) 1264;

A. Salam, Elementary Particle Theory, ed. by N. Svartholm, Stockholm (1968), 367.

[2] P. Tipto (CDF Collab.) and N.Hadley (D0 Collab.), Plenary talks at the XVI Int. Symp. on Lepton-Photon Interactions, Cornell Univ., Ithaka, N.Y., U.S.A., Aug 10-15, 1993.

[3] W. Hollik, Plenary talk at the XVI Int. Symp. on Lepton-Photon Interactions, Cornell Univ., Ithaka, N.Y., U.S.A., Aug 10-15, 1993;

J. Le Francois, Plenary talk at the Int. Europhys. Conf. on High En.Phys. Marseille, July 22-28, 1993.

[4] C. P. Yuan, Phys. Rev. D41 (1990) 42;

T. Moers et al., Aachen ECFA Workshop, 1990, p.418;

G. V. Jikia and S.R.Slabospitsky, Phys. Lett. B295 (1992) 136.

[5] S. Ambrosanio and B. Mele, Preprint Rome -979 -1993.

[6] M. Katuya, J. Morishita, T. Munehisa and Y. Shimizu, Prog. Theor. Phys 75 (1986) 92.

[7] G. Jikia, Nucl. Phys. B374 (1992) 83;

E. Yehudai, S. Godfrey and K. A. Peterson, Preprint Fermilab -Conf -93224 (1993).

[8] M. Kobayashi and T. Maskawa, Progr. Theor. Phys. 49 (1973) 652.

[9] M. Raidal and R. Vuopionperä, Phys. Lett. B318 (1993) 237.

[10] O. Panella, G. Pancheri and Y. N. Srivastava, Phys. Lett. B318 (1993) 241. 
[11] E. E. Boos et al., in: Proc. of the XXVIth Recontre de Moriond, ed. by J. Tran Than Van, Edition Frontiers, 1991, p. 501;

E. E. Boos et al., in: Proc. of the Second Int. Workshop on Software Engineering, ed. by D. Perred-Gallix, World Scientific, 1992, p. 665.

[12] T. Ishikawa et al., GRACE manual, KEK report 92-19, 1992.

[13] S. Kawabata, Comp. Phys. Commun. 41 (1986) 127.

[14] E. E. Boos et al., KEK Preprint 92-47, 1992.

[15] A. Aeppli, F. Cuypers and G. J. van Oldenborgh, Phys. Lett. B314 (1993) 413.

[16] C. F. Weizsäcker, Z. Phys. 88 (1934) 612;

E. J. Williams, Phys. Rev. 45 (1934) 729.

[17] S. Frixione, M. Mangano, P. Nason and G. Ridolfi, Phys. Lett. B319 (1993) 339. 


\section{Figure Caption}

Fig. 1: Feynman diagrams for the single top quark production in the reaction $e^{+} e^{-} \longrightarrow e \nu t b$. Diagrams involving the Higgs boson are omitted due to the smallness of the Higgs-electron coupling.

Fig. 2: Total cross sections for the reaction $e^{+} e^{-} \longrightarrow e^{+} \nu_{e} \bar{t} b\left(e^{-} \bar{\nu}_{e} t \bar{b}\right)$ as function of the top quark mass for $\sqrt{s}=170,190$ and $210 \mathrm{GeV}$. The solid line corresponds to the complete set of diagram calculations, the dashed line to the photon exchange diagram approximation and the dotted line to the Weizsäcker-Williams approximation.

Fig. 3: Total cross sections in the four photon exchange diagram approximation as a function of the positron angular cut for $m_{\text {top }}=140 \mathrm{GeV}$ at $\sqrt{s}=190 \mathrm{GeV}$ in the unitary gauge. The dotted lines show the results for each of the diagrams (denoted as (A), (B), (C) and (D) in Fig. 1) squared, while the solid line corresponds to their coherent sum. 


\begin{tabular}{|c|c|c|c|c|}
\hline $\begin{array}{c}\sqrt{s} \\
\mathrm{GeV}\end{array}$ & $\begin{array}{c}m_{\text {top }} \\
\mathrm{GeV}\end{array}$ & $\begin{array}{c}\text { full set } \\
\mathrm{pb}\end{array}$ & $\begin{array}{c}4 \text { diagrams } \\
\mathrm{pb}\end{array}$ & $\begin{array}{c}\text { W-W approx. } \\
\mathrm{pb}\end{array}$ \\
\hline \multirow{3}{*}{170} & 130 & $3.6410^{-6}$ & $3.5310^{-6}$ & $4.5310^{-6}$ \\
& 160 & $1.4010^{-9}$ & $1.4410^{-9}$ & $1.9810^{-9}$ \\
\hline \multirow{7}{*}{190} & 130 & $1.6210^{-5}$ & $1.5710^{-5}$ & $1.9910^{-5}$ \\
& 160 & $8.8210^{-7}$ & $8.6110^{-7}$ & $1.1210^{-6}$ \\
& 180 & $1.2010^{-9}$ & $1.2110^{-9}$ & $1.6610^{-9}$ \\
\hline \multirow{2}{*}{210} & 130 & $4.2810^{-5}$ & $4.0610^{-5}$ & $5.0710^{-5}$ \\
& 160 & $6.8810^{-6}$ & $6.7010^{-6}$ & $8.5810^{-6}$ \\
& 180 & $7.5210^{-7}$ & $7.3010^{-7}$ & $9.5410^{-7}$ \\
\hline
\end{tabular}

Table 1: Cross sections for the reaction $e^{+} e^{-} \longrightarrow e^{+} \nu_{e} \bar{t} b\left(e^{-} \bar{\nu}_{e} t \bar{b}\right)$ for the complete set of diagrams, the four $t$-channel photon exchange diagrams and the Weizsäcker-Williams approximation. 


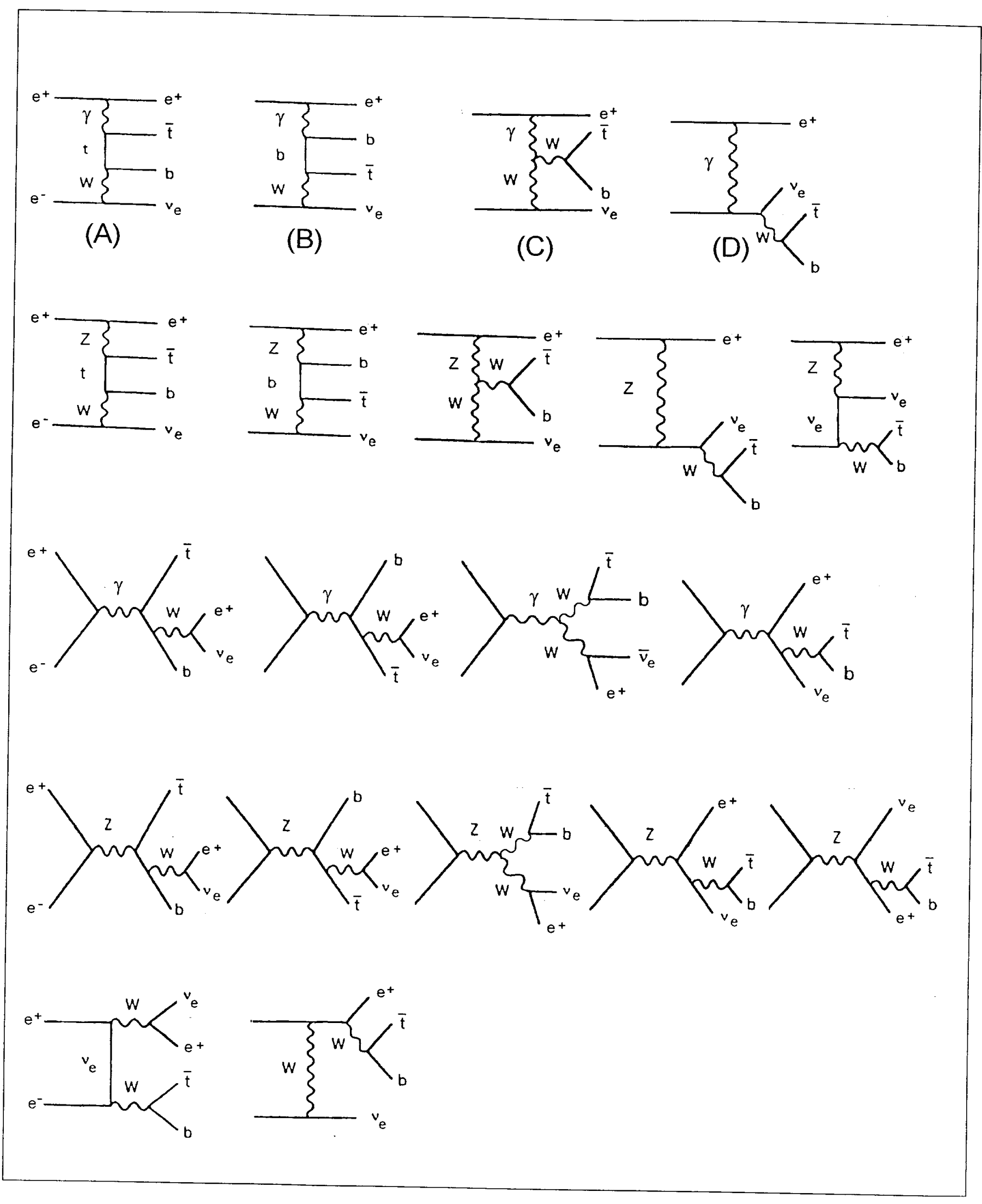

Figure 1: 


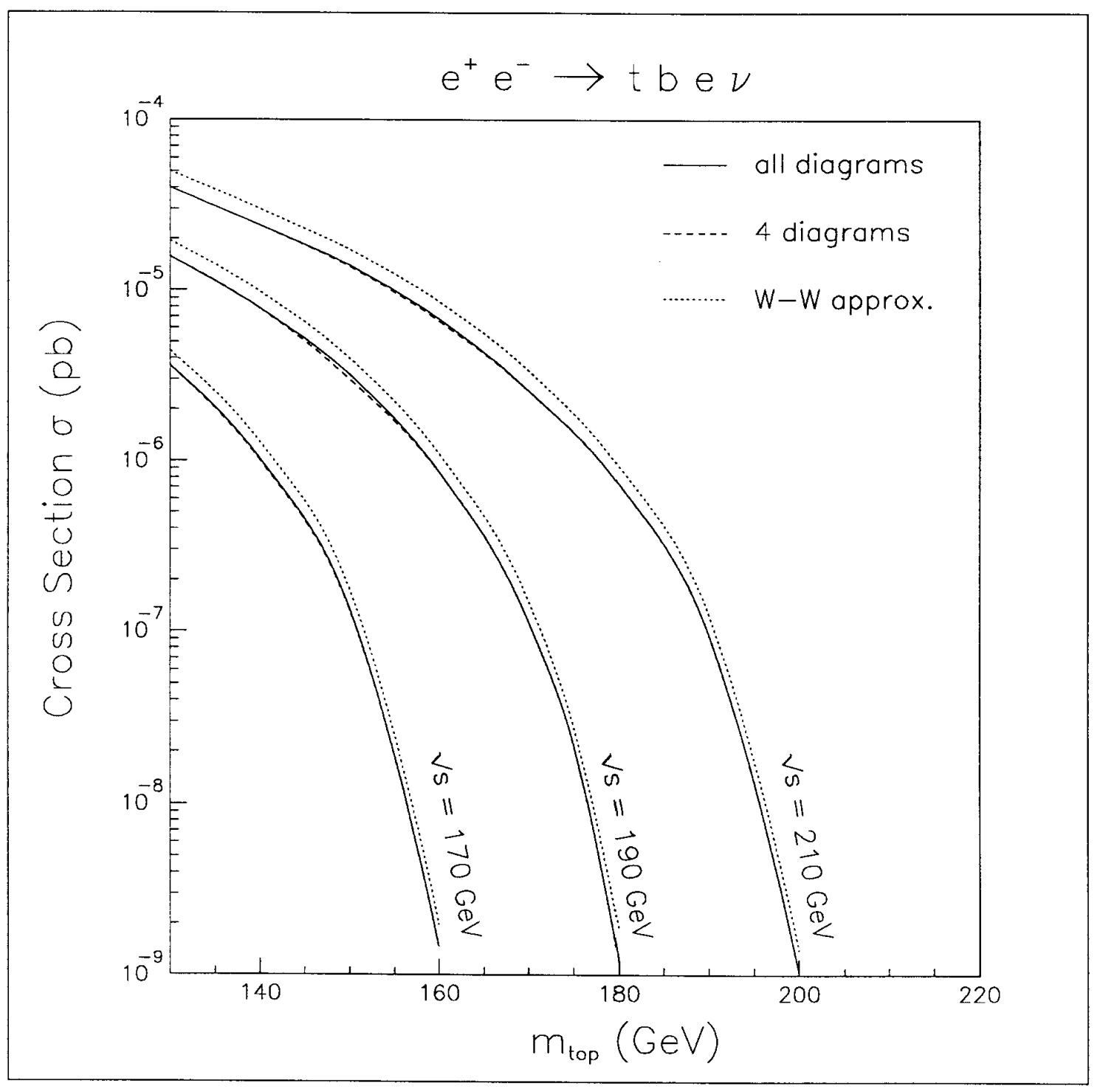

Figure 2: 


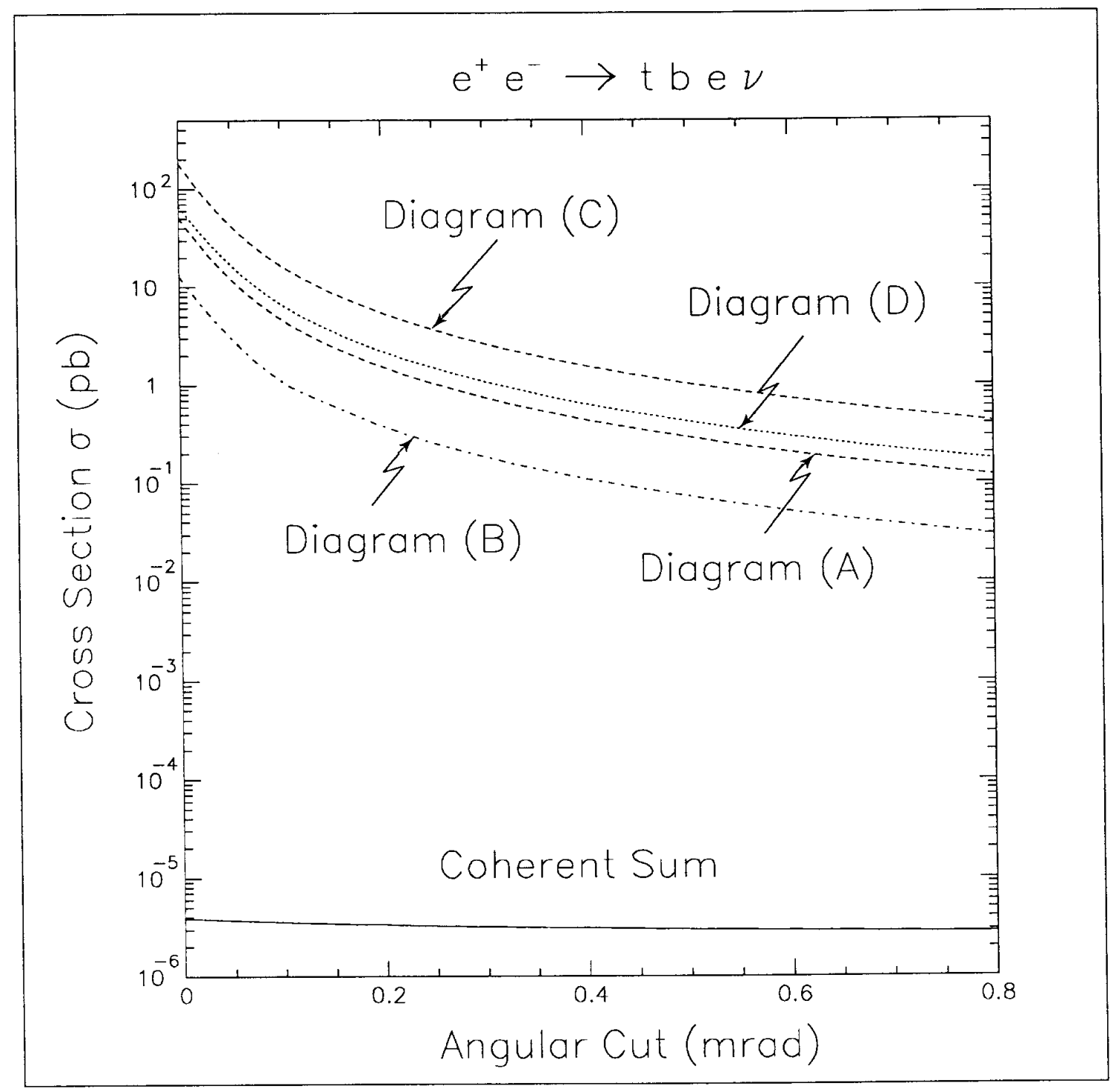

Figure 3: 